\title{
Unterscheiden sich die Einstellungen von MCl-Patienten einer Gedächtnissprechstunde von denen einer Versorgerpraxis?
}

\section{Differences in Attitudes of Patients with Mild Cognitive Impairment Towards Early Diagnosis at a University Clinic and a Specialist Physician's Office}

\author{
Autoren \\ J. Bohlken¹, T. Köbe², L. M. Dietz ${ }^{2}$, M. A. Rapp ${ }^{3}$, T. Kohlmann ${ }^{4}$ A. Flöel ${ }^{2}$ \\ Institute \\ BVDN, Praxis Bohlken, Berlin \\ 2 NeuroCure Clinical Research Center, Charité Universitätsmedizin Berlin \\ Kognitionswissenschaften, Sozial- und Präventivmedizin, Potsdam \\ ${ }^{4}$ Community Medicine, Universität Greifswald
}

Schlüsselwörter
Demenz
Füherkennung
Patienteneinstellun
Liquorpunktion
Key words
dementia
early diagnosis
attitudes
lumbar puncture

\section{Bibliografie}

Dol http://dx.doi.org/ 10.1055/s-0041-108097

Fortschr Neurol Psychiatr 2015; 83: 563-567 @ Georg Thieme Verlag KG Stuttgart · New York . ISSN 0720-4299

\section{Korrespondenzadresse}

Prof. Michael Armin Rapp

Kognitionswissenschaften,

Sozial- und Präventivmedizin

Am Neuen Palais 10

14469 Potsdam

mrapp@uni-potsdam.de

\section{Zusammenfassung \\ $\nabla$}

Ziel der Studie: Untersuchung der Einstellungsmuster von Patienten mit leichter kognitiver Störung hinsichtlich Diagnostik in unterschiedlichen Versorgungssituationen.

Methodik: Vergleichende Befragung von $129 \mathrm{~Pa}-$ tienten in einer Universitätsambulanz $(\mathrm{n}=38)$ und einer Facharztpraxis $(n=91)$ über ihre Einstellung zur Demenz-Frühdiagnostik und Bereitschaft zur Liquordiagnostik.

Ergebnisse: Die Bereitschaft zur Liquordiagnostik war in der Universitätsambulanz höher als in der Facharztpraxis $(p=0,040)$, die generelle Bereitschaft zu Frühdiagnostik vergleichbar hoch.

Schlussfolgerung: Unterschiedliche Einstellungen von Patienten mit leichter kognitiver Störung sollten in unterschiedlichen Versorgungssituationen Berücksichtigung finden.

\section{Abstract \\ $\nabla$}

Aim: To examine the attitude of patients with mild cognitive impairment to diagnostics under different healthcare settings.

Methodology: A comparative survey was carried out of 38 patients at a university outpatient clinic and 91 patients at a specialist practice with regard to their attitudes towards early diagnosis of dementia and willingness to undergo CSF analysis. Results: Willingness to undergo CSF analysis was higher among the patients at the university outpatient unit than those at the specialist practice $(p=0.040)$, and willingness to undergo early diagnosis was comparable high in both groups.

Conclusion: Different attitudes of patients with mild cognitive impairment should be reflected in different healthcare settings.

\section{Einleitung}

\section{$\nabla$}

In einer alternden Gesellschaft wird es gesundheitspolitisch entscheidend sein, Strategien zu entwickeln, die den strukturellen und funktionellen Abbau des menschlichen Gehirns im Alter verlangsamen können. Während es für das Frühstadium der Alzheimer-Demenz zugelassene und in Leitlinien empfohlene Medikamente gibt [1], stehen Strategien für leichte kognitive Störungen (engl. mild cognitive impairment, MCI) aus [2 - 4]. Für die Entwicklung neuer medikamentöser Therapien werden krankheitsbezogene Stratifizierungen der Patienten mittels Demenz-spezifischer Biomarker als wesentlich angesehen. Diese sollen erlauben, diejenigen MCI-Patienten zu identifizieren, deren kognitive Defizite mit hoher Wahrscheinlichkeit auf einer Alzheimer-Krankheit beruhen und die wahrscheinlich in Zukunft eine Alzheimer-Demenz entwickeln werden $[5,6]$. Bisher ist eine Früherkennung der Alzheimer-Demenz im Stadium des MCI nur näherungsweise durch die Bestimmung verschiedener Biomarker möglich [5, 7-9]. Zwei davon werden im Liquor nachgewiesen (CSF Aß42; Tau, p-Tau), drei basieren auf der Gehirnbildgebung (Hypometabolismus im FDG-PET [10], AmyloidPET [11]; Atrophiemuster im strukturellen MRT [12]). Auch wenn die Validität dieser Biomarker in Autopsiestudien grundsätzlich etabliert wurde, so ist eine sichere Voraussage der Entwicklung einer Alzheimer-Demenz im MCI Stadium nicht möglich $[8,13]$. Die Voraussage der zeitlichen Entwicklung des Auftretens der Krankheitssymptomatik (klinische Demenz) sowie des Schweregrads der Erkrankung ist nur eingeschränkt gewährleistet $[7-9,13]$. Die PET-Untersuchung umfasst die Applikation von radioaktiver Strahlung und ist damit insbesondere für Wiederholungsmessungen wenig geeignet; das Amyloid-PET ist nur an wenigen Standorten verfügbar. Die Messung von Atrophiemustern im zerebralen MRT ermöglicht erst relativ spät im Krankheitsverlauf eine prädiktive Aussage [14]. 
Schließlich ist die Liquorpunktion (LP) eine invasive Maßnahme, die neben häufig auftretenden Kopfschmerzen auch mit selten auftretenden, aber potenziell gravierenden Risiken wie einer Infektion des Nervensystems, Blutungen oder Liquorlecks verbunden ist. In der gegenwärtigen Routineversorgung spielen die neuropsychologische Testung und die MRT-Bildgebung eine wichtige, wenn auch zu wenig genützte Rolle bei der Demenz-Diagnostik [15]. Spezifische PET-Unterschungen sind keine Kassenleistungen und demenzspezifische Liquoruntersuchungen sind im ambulanten Bereich zwar Kassenleistungen, werden aber bei Demenzen selten eingesetzt. Diese Untersuchungen werden in der Regel in universitären Gedächtnissprechstunden angeboten.

Über die subjektive Einstellung von Patienten mit MCI gegenüber frühdiagnostischen Verfahren ist wenig bekannt. Vergleichende Untersuchungen an Patienten von neuropsychiatrischen Facharztpraxen und Universitätsambulanzen liegen nicht vor.

Primäres Ziel dieser Studie war es zu untersuchen, ob Patienten aus einer universitären Gedächtnissprechstunde (Uni-GS) eine höhere Bereitschaft zur Durchführung einer LP aufweisen als MCI-Patienten aus einer Facharztpraxis (FA-Praxis). Als abhängige Variable wurde der Prozentanteil der insgesamt jeweils befragten Patienten festgelegt, die bereit sind, eine LP durchführen zu lassen. Des Weiteren sollte erfragt werden, ob Unterschiede in der Bereitschaft zur generellen Frühdiagnostik bestehen. Im Rahmen einer sekundären Analyse wurde über die gesamte Stichprobe hinweg untersucht, ob diese Zielvariablen von Alter, Geschlecht, Bildung, Einkommen, Wohnsituation, kognitivem Status und Depressivität beeinflusst werden.

\section{Methoden}

$\nabla$

Die Studie wurde in der neurologischen Gedächtnissprechstunde des Universitätsklinikums Charité Mitte Berlin (Uni-GS; Prof. Dr. A. Flöel) sowie einer neurologischen Facharztpraxis in Berlin (FAPraxis; Dr. J. Bohlken) durchgeführt. Die FA-Praxis rekrutierte die Patienten im Rahmen der Routineversorgung eines Berliner Bezirks überwiegend durch hausärztliche Zuweisung. Die Uni-GS rekrutiert ihre Patienten im Rahmen von fachärztlichen $\mathrm{Zu}$ weisungen bei unklaren diagnostischen Zuständen sowie im Rahmen von Studienausschreibungen. Diese Pilotstudie wurde in beiden Einrichtungen im Rahmen des normalen Versorgungsalltags durchgeführt. Vor Studienteilnahme wurden alle Patienten mündlich und schriftlich über die Studieninhalte informiert und unterzeichneten eine Einverständniserklärung zur Teilnahme. Die Studie wurde von der Ethikkommission der Universität Greifswald und von der Ethikkommission der Charité Universitätsmedizin Berlin genehmigt und entspricht der Erklärung von Helsinki.

Insgesamt wurden im Zeitraum vom Juli 2013 bis Mai 2014129 konsekutive MCI-Patienten im Alter von 50-90 Jahren rekrutiert. Die Bereitschaft zur Teilnahme an der Befragung lag in der FA-Praxis bei $100 \%$, in der Uni-GS bei $95 \%$. Die MCI-Diagnose wurde wie folgt operationalisiert: a. subjektive Gedächtnisverschlechterungen, mitgeteilt durch den Patienten oder einen Angehörigen, b. objektive Gedächtnisschwierigkeiten, die mindestens 1,5 Standardabweichungen unter geschlechts-, alters- und bildungsadjustierten Normwerten fallen (Logical Memory delayed-recall score der Wechsler Memory Scale III; Uni-GS; Demtect $\geq 9$ Punkte; FA-Praxis [16]), c. relativ normale Leistungen in anderen kognitiven Domänen, d. keine Einschränkungen bei sonstigen Alltagsaktivitäten, e. keine manifeste Demenz. Aus den Krankenakten wurden die Sozialdaten entnommen (Alter, Geschlecht, Anzahl der Schuljahre), das Ergebnis des aktuellen Mini-Mental-Status-Tests (MMST) [17] sowie das Ergebnis der institutionsspezifischen Depressions-Selbstbeurteilungs-Skala Uni-GS: Beck-Depressions-Inventar (BDI) [18] und FA-Praxis: Geriatrische Depressionsskala (GDS) [19].

Das Interview folgte einem standardisierten Fragebogen mit insgesamt 15 Fragen. Dazu gehörten Fragen zur generellen Bereitschaft zur Frühdiagnostik („Würden Sie sich untersuchen lassen, um Ihr Demenzrisiko abzuschätzen, auch wenn es keine Therapie gibt?“) und zur spezifischen Bereitschaft zur Liquorpunktion („Würden Sie einer Untersuchung des Nervenwassers zustimmen, die möglicherweise schmerzhafter sein kann als eine Blutentnahme“). Es wurden jeweils drei Antwortmöglichkeiten vorgegeben: ja, nein, weiß nicht. Die Fragefolge wurde durch eine kurze Falldarstellung eingeleitet, in der die Problematik der Frühdiagnostik (Untersuchung des Nervenwassers, Mitteilung eines erhöhten Erkrankungsrisikos, Zeitrahmen, Fehlen einer medikamentösen Therapie) aus Blickrichtung des Standardversorgers (J.B.) dargestellt wurde, der typischerweise nicht die in universitären Gedächtnissprechstunden vorgehaltenen Methoden einer biomarkerorientierten Diagnostik vorhält. (s. Anmerkung $^{1}$ ). Eine spezifische Aufklärung zur Diagnostik erfolgte vor der Befragung nicht.

\section{Statistische Analyse \\ $\nabla$}

Die Gegenüberstellung der demografischen Parameter wie Alter, Geschlecht und Bildung zwischen den Institutionen Uni-GS und FA-Praxis erfolgte entsprechend gekennzeichnet mittels eines ungepaarten t-Tests (normalverteilte Daten), Mann-Whitney-UTests (nicht normalverteilte Daten) oder Pearson Chi²-Tests (für kategoriale Daten). Die Hypothese, dass sich MCI-Patienten aus einer Uni-GS hinsichtlich der Bereitschaft zur Liquorpunktion oder Frühdiagnostik von MCI-Patienten aus einer FA-Praxis unterscheiden, wurde in einem Gruppenvergleich mittels Pearson $\mathrm{Chi}^{2}$-Test und zusätzlich in einer binären logistischen Regression unter Einbeziehung von Alter, Geschlecht und Bildung als Kovariate getestet. Die Gesamtstichprobe betreffend wurden mögliche Einflussfaktoren wie Alter, Geschlecht, Bildung, Einkommen, Wohnsituation, MMST und Depressivität auf die zu untersuchenden Variablen mittels Chi²-Test oder bivariater Korrelation nach Pearson auf Signifikanz untersucht. Die Software SPSS 20 (PASW, SPSS; IBM, Armonk, NY) wurde für die statistische Analyse verwendet. Die Normalverteilung der Daten wurde mit dem Kolmogorov-Smirnov-Test geprüft und die Signifikanzschwelle bei $\mathrm{p}<0,05$ (zweiseitig) festgelegt.

\section{Ergebnisse \\ $\nabla$}

Insgesamt wurden 129 Patienten mit MCI befragt (71 Frauen, Durchschnittsalter 70,1 Jahre), wovon $60 \%$ weniger als 10 Jahre

\footnotetext{
${ }^{1}$ Anm. Erläuternde Falldarstellung zu Beginn des Interviews: „Herr Meier ist 65 Jahre alt. Er stellte sich wegen leichter Gedächtnisstörungen in einer Universitätsklinik vor. Dort wurde neben vielen anderen Untersuchungen auch das Nervenwasser untersucht. Als Ergebnis wurde ihm mitgeteilt, dass er keine Demenz habe, aber dass ein hohes Risiko besteht, in den nächsten 5 Jahren eine Demenz zu bekommen. Leider gebe es gegenwärtig keine medikamentöse Hilfe. Herr Meier ist nun in großer Sorge.“
} 
die Schule besuchten. Das Haushaltsnettoeinkommen pro Person betrug bei $50 \%$ weniger als $1000 €$ im Monat. $75 \%$ lebten mit Partner oder einer anderen Person zusammen, 25\% lebten allein. Der Durchschnittswert des MMST betrug 28,7 Punkte und 32\% wiesen in den Selbstbeurteilungsskalen (BDI, GDS) depressionstypische Werte auf. Im Vergleich zur Stichprobe der FA-Praxis ( $n=91,55$ Frauen, Durchschnittsalter 70 Jahre) wies die Stichprobe der Uni-GS ( $n=38,16$ Frauen, Durchschnittsalter 70 Jahre) einen höheren Anteil an Männern (Pearson $\left.\mathrm{X}_{(1)}=3,641, \mathrm{p}=0,056\right)$ und eine höhere Schulbildung (Pearson $\mathrm{X}^{2}{ }_{(1)}=11,687, \mathrm{p}=0,001$ ) auf. Keine Unterschiede fanden sich hinsichtlich des Alters, des Einkommens, der Wohnsituation, der kognitiven Einschränkungen im MMST und der depressiven Symptomatik ( $\bullet$ Tab. 1).

Die Bereitschaft zur Teilnahme an einer Demenzrisiko-Untersuchung lag für die Gesamtstichprobe bei $67 \%$ (Uni-GS=58\%, FAPraxis $=71 \%$ ). Die Bereitschaft zur LP lag bei $34 \%$, wobei Patienten der Uni-GS (47\%) signifikant häufiger bereit waren, eine LP durchführen zu lassen, als Patienten der FA-Praxis (29\%) $\left(\mathrm{X}_{(1)}=4,214, \mathrm{p}=0,040\right)$ ( $\bullet$ Tab. 2). Eine Korrektur für Alter, Geschlecht und Bildung mindert diesen Effekt, sodass nur noch ein Trend $\mathrm{zu}$ verzeichnen war (Wald $\mathrm{X}_{(1)}=2,681, \mathrm{p}=0,102$ ). Alter, Geschlecht, Bildung, Einkommen, Wohnsituation, MMST und Depressivität hatten keinen Einfluss auf die Bereitschaft, sich untersuchen zu lassen $(\mathrm{p}>0,106)$.

\section{Diskussion}

$\nabla$

In einer standardisierten Befragungsstudie wurden erstmals vergleichend Einstellungsmuster von Patienten mit leichten kognitiven Störungen in zwei unterschiedlichen Versorgungsinstitutionen, nämlich einer universitären Gedächtnissprechstunde (Uni-GS) und einer fachärztlichen Versorgerpraxis mit Schwerpunkt Demenz (FA-Praxis), untersucht.

Die Bereitschaft zu einer Untersuchung des Nervenwassers (Liquorpunktion) war entsprechend unserer primären Hypothese in der universitären Stichprobe mit einer Zustimmungsrate von $47 \%$ höher als in der Facharztstichprobe, wo sie bei $29 \%$ lag. Andere Studien [20] berichten deutliche höhere Zustimmungsraten von bis zu 70\%. Allerdings wurden in den USA im hausärztlichen Bereich ebenfalls geringe Bereitschaften für weit weniger invasive Untersuchungsmethoden gefunden [21]. Angesichts kontroverser Debatten über die Einführung von Liquoruntersuchungen in die Routineversorgung $[22,23]$ bleibt aber festzuhalten, dass mehr als ein Drittel bis die Hälfte der Studienteilnehmer die Liquoruntersuchungen wünschen.

Bezüglich der Bereitschaft zur generellen Frühdiagnostik konnten wir keine wesentlichen Unterschiede zwischen der Uni-GS und der FA-Praxis feststellen. Auch konnten keine signifikanten Einflussvariablen auf diese Bereitschaft identifiziert werden. Im Vergleich zur Befragung der Allgemeinbevölkerung mit einer Zustimmungsrate zur Frühdiagnostik von $88 \%$ [24] war die Bereitschaft zur Frühdiagnostik in unserer Studie mit 67\% geringer. Andere

\begin{tabular}{|c|c|c|c|c|}
\hline MCI-Patienten & $\begin{array}{l}\text { Gesamt } \\
(n=129)\end{array}$ & $\begin{array}{l}\text { Uni-GS } \\
(n=38)\end{array}$ & $\begin{array}{l}\text { FA-Praxis } \\
(\mathrm{n}=91)\end{array}$ & $\begin{array}{l}\text { P } \\
\text { Uni-GSS vs FA }\end{array}$ \\
\hline \multicolumn{5}{|l|}{ Alter } \\
\hline Jahre & 70,1 & 70,3 & 70,0 & \multirow[t]{2}{*}{$0,853^{1}$} \\
\hline [MW s (min-max)] & $8,5(48-88)$ & $7,9(52-82)$ & $8,7(48-88)$ & \\
\hline \multicolumn{5}{|l|}{ Geschlecht } \\
\hline weiblich [\% (n)] & $55(71)$ & $42(16)$ & $60,4(55)$ & $0,056^{2}$ \\
\hline \multicolumn{5}{|l|}{ Bildung (Schule) } \\
\hline$\leq 10$ Jahre $[\%(n)]$ & $60(77)$ & $37(14)$ & $69(63)$ & \\
\hline$>10$ Jahre [\% (n)] & $40(52)$ & $63(24)$ & $31(28)$ & $0,001^{2}$ \\
\hline \multicolumn{5}{|l|}{ Einkommen } \\
\hline$<1000 €[\%(n)]$ & $50(65)$ & $63(24)$ & $45(41)$ & \\
\hline$>1000 €[\%(n)]$ & $50(64)$ & $37(14)$ & $55(50)$ & $0,061^{2}$ \\
\hline \multicolumn{5}{|l|}{ Wohnsituation } \\
\hline allein [\% (n)] & $25(32)$ & $24(9)$ & $25(23)$ & \\
\hline mit Partner [\% (n)] & $75(97)$ & $76(29)$ & $75(68)$ & $0,849^{2}$ \\
\hline \multicolumn{5}{|c|}{ Klinische Parameter } \\
\hline MMST [score] ${ }^{3}$ & 28,7 & 28,6 & 28,6 & \\
\hline [MW s (min-max)] & $1,4(24-30)$ & $1,2(26-30)$ & $1,5(24-30)$ & $0,982^{1}$ \\
\hline \multicolumn{5}{|l|}{$\mathrm{BDI} \mathrm{GDS}^{4}$} \\
\hline$\leq 10 / \leq 4[\%(n)]$ & $68(77)$ & $72(23)$ & $66(54)$ & \\
\hline$>10 />4[\%(n)]$ & $32(37)$ & $28(9)$ & $34(28)$ & $0,537^{2}$ \\
\hline \multicolumn{5}{|c|}{$\begin{array}{l}{ }^{1} \text { ungepaarter t-Test. } \\
{ }^{2} \text { chi }^{2} \text {-Test. } \\
\text { fehlende Antworten: } \\
\left.{ }^{3} \mathrm{n}(\text { Uni-GS })=32, \mathrm{n} \text { (FA-Praxis }\right)=86 . \\
\left.{ }^{4} \mathrm{n}(\text { Uni-GS })=32, \mathrm{n} \text { (FA-Praxis }\right)=82 .\end{array}$} \\
\hline
\end{tabular}

Tab. 1 Stichprobe MCl-Patienten gesamt und nach Institution (Uni-GS/FA-Praxis).

\begin{tabular}{|lllll|}
\hline Bereitschaft zur & $\begin{array}{l}\text { Gesamt } \\
(\mathbf{n = 1 2 9 )}\end{array}$ & $\begin{array}{l}\text { Uni-GS } \\
(\mathbf{n}=\mathbf{3 8})\end{array}$ & $\begin{array}{l}\text { FA-Praxis } \\
(\mathbf{n}=\mathbf{9 1})\end{array}$ & $\begin{array}{l}\text { Uni-GS vs FA-Praxis } \\
\mathrm{X}^{\mathbf{2}} \mathbf{p} \mathbf{p}\end{array}$ \\
\hline Frühdiagnostik & & & & \\
\hline Frühdiagnostik: JA [\%(n)] & $67(87)$ & $58(22)$ & $71(65)$ & 2,$236 ; 0,135$ \\
\hline Liquorpunktion: JA [\%(n)] & $34(44)$ & $47(18)$ & $29(26)$ & 4,$214 ; 0,040$ \\
\hline
\end{tabular}

Tab. 2 Bereitschaft zur Frühdiagnostik und Liquordiagnostik. 
Studien berichten positive Zusammenhänge einer erhöhten Bereitschaft zur Frühdiagnostik mit höherem Lebensalter [25 - 27] und besserem kognitivem Status [25]. Allerdings konnte jüngst in einem Prä-post-Design gezeigt werden, dass die Zustimmungsbereitschaft sinkt, wenn eine Bedenkzeit eingeräumt wird, um die Konsequenzen der Frühdiagnostik abzuwägen [28]. Wir haben deshalb in unserer Befragungsstudie eine Falldarstellung vorangestellt, um einen vergleichbaren Wissensstand bei den Befragten zu gewährleisten. Möglicherweise hat diese Falldarstellung zu den vergleichsweise niedrigen Zustimmungsraten geführt.

Insgesamt erscheint aber die Bereitschaft zur Frühdiagnostik mit über zwei Dritteln in dieser und anderen Studien [24-26] angesichts der eingeschränkten Behandlungsmöglichkeiten erstaunlich hoch. Insbesondere für den hausärztlichen Bereich wird vor den unbeabsichtigten Folgen einer forcierten biomarkerorientierten Diagnostik gewarnt [29]. Bedenken sind hier das Fehlen einer medizinischen Behandlungsmöglichkeit [3], die Möglichkeit der diagnostischen Fehleinschätzung [30], die beschränkte prognostische Aussagekraft unter Routinebedingungen [29] sowie unbeabsichtigte Folgen der Diagnosemitteilung wie z. B. Stigmatisierung und Auslösen von z. B. depressiven Anpassungsstörungen [29]. Demgegenüber stehen aber Vorteile hinsichtlich einer optimierten internistischen Behandlung, einer frühen Aktivierung von sozialen Hilfesystemen, der verzögerten Aufnahme in ein Pflegeheim und der Möglichkeit, ohne gesetzlichen Beistand in einem kognitiv nur wenig eingeschränkten Zustand autonom Lebensentscheidungen zu treffen [24, 31, 32].

Folgende methodische Einschränkungen sollten bei der Bewertung der Ergebnisse berücksichtigt werden: Diese Pilotstudie wurde auf Anregung von J. B. ohne weitere Forschungsförderung in beiden Einrichtungen im Rahmen des normalen Versorgungsalltags durchgeführt. Möglicherweise ist dadurch der universitären Sichtweise nicht genügend Rechnung getragen worden. Beide Stichproben berücksichtigen Patienten, die sich wegen Gedächtnisstörungen vorstellten, ohne dass bereits ein Demenzsyndrom mit offensichtlichen Einschränkungen der Alltagsfähigkeiten vorlag. Es handelt sich also um eine besonders aufmerksame Patientengruppe. Andererseits kann angesichts des relativ hohen Anteils an MCI-Patienten mit relevanten depressiven Symptomen, der mit internationalen Studien durchaus vergleichbar ist [33], ebenso von einem deutlichen Leidensdruck ausgegangen werden. Die universitäre Stichprobe konnte nur über einen kürzeren Zeitraum rekrutiert werden und ist deshalb kleiner. Diesem Umstand ist es geschuldet, dass die universitäre Stichprobe kleiner ist und einen hohen Anteil an Männern aufweist, und dies stellt in sich eine methodische Einschränkung dar, die auch einen Einfluss auf die Stabilität der Ergebnisse gegenüber Kovariaten gehabt haben dürfte. Weiterhin wurden unterschiedliche Methoden bei der neuropsychologischen Testung (in der Praxis nur DemTect) und der Depressionsmessung (GDS gegenüber BDI) verwendet. Validierungsstudien zeigen aber hier gute Übereinstimmungswerte [16]. Aus Gründen der Zeitersparnis wurde auf ein Interview mit offenen Fragen verzichtet und ein Verfahren mit einfachen Antwortvorgaben gewählt. Bestimmte inhaltliche Aspekte wurden nicht berücksichtigt, dazu gehören das Vorwissen der Patienten, das Krankheitsverhalten, die Persönlichkeit und die Besonderheiten des Arzt-Patienten-Verhältnisses. Mit Blickrichtung auf die Versorgungsrealität wurde auf eine Erläuterung und Befragung bezüglich der nuklearmedizinischen Diagnostik beispielsweise mit Amyloid-PET verzichtet. Schließlich entstand die Studie im Rahmen einer Rekrutierungsphase einer laufenden Interventionsstudie, bei der es um die Un- tersuchung des Einflusses von Nahrungsergänzungsmitteln und sportlicher Aktivität ging. Die Fragen zur Veränderungsbereitschaft des Lebensstils wurden aus Platzgründen nicht dargestellt. Zwischen den beiden Einrichtungen fanden sich keine Unterschiede hinsichtlich der Bereitschaft zu geistiger und sportlicher Aktivitätssteigerung sowie zur Ernährungsumstellung.

\section{Konsequenzen für Klinik und Praxis}

- Die Ergebnisse dieser Pilotstudie bei Patienten mit leichten kognitiven Störungen belegen, dass die Bereitschaft zu einer invasiveren Frühdiagnostik (Liquorpunktion) in einer universitätsklinischen Gedächtnissprechstunde höher ist als in einer Facharztpraxis.

- Die Bereitschaft zur Frühdiagnostik unterscheidet sich in beiden Einrichtungen nicht wesentlich.

- Unterschiedliche Einstellungsmuster zur Liquorpunktion sollten in Abhängigkeit von unterschiedlichen Versorgungssituationen Berücksichtigung finden. Wenn insbesondere neurologische Facharztpraxen Liquorpunktionen auch bei MCI-Patienten durchführen sollten, benötigen die Patienten dann eine intensivere Aufklärung.

Interessenkonflikt: J. Bohlken erhielt finanzielle Zuwendungen für Studiendurchführung und Vorträge von der Firma Willmar Schwabe sowie Zuwendungen vom BVDN. M. A. Rapp erhielt in den letzten fünf Jahren finanzielle Zuwendungen für Vorträge von den Firmen Willmar Schwabe, Merz, Johnson \& Johnson sowie GlaxoSmithKline.

\section{Literatur}

1 Rountree SD, Atri A, Lopez OL et al. Effectiveness of antidementia drugs in delaying Alzheimer's disease progression. Alzheimers Dement 2013; 9: $338-345$

2 Deutsche Gesellschaft für Psychiatrie, Psychotherapie und Nervenheilkunde (DGPPN), and Deutsche Gesellschaft für Neurologie. eds. Diagnose- und Behandlungsleitlinie Demenz. Heidelberg: Springer Medizinverlag; 2010

3 Gertz H, Kurz A. Diagnose ohne Therapie. Frühdiagnostik der Alzheimer-Krankheit im Stadium der leichten kognitiven Beeinträchtigung. Nervenarzt 2011; 82: 1151 - 1159

4 Jessen $F$. Therapy for patients with dementia: Treatment strategies in the elderly. Internist (Berl) 2014; 55: 769-774

5 Dubois B, Feldman HH, Jacova C et al. Revising the definition of Alzheimer's disease: a new lexicon. Lancet Neurol 2010; 9: 1118-1127

6 Jack CR, Knopmann DS et al. Hypothetical model of dynamic biomarkers of the Alzheimer's pathological cascade. Lancet Neurology 2010; 9: $119-128$

7 Sperling RA, Aisen PS, Beckett LA et al. Toward defining the preclinical stages of Alzheimer's disease: recommendations from the National Institute on Aging-Alzheimer's Association workgroups on diagnostic guidelines for Alzheimer's disease. Alzheimers Dement 2011; 7: 280 - 292

8 Jessen F, Wiese B, Bachmann C. German Study on Aging, Cognition and Dementia in Primary Care Patients Study Group. et al. Prediction of dementia by subjective memory impairment: effects of severity and temporal association with cognitive impairment. Arch Gen Psychiatry 2010; 67: 414-422

9 Albert MS et al. The diagnosis of mild cognitive impairment due to Alzheimer's disease: Recommendations from the National Institute on Aging-Alzheimer's Association workgroups on diagnostic guidelines for Alzheimer's disease. Alzheimer`s \& Dementia 2011; 7: 270-279

10 Jagust WJ, Bandy D, Chen K. Alzheimer's Disease Neuroimaging Initiative. et al. The Alzheimer's Disease Neuroimaging Initiative positron emission tomography core. Alzheimers Dement 2010; 6: 221 - 229

11 Nordberg A. Amyloid plaque imaging in vivo: current achievement and future prospects. Eur J Nucl Med Mol Imaging 2008; 35 (Suppl 1): S46-S50 
12 Dickerson $B C$, Wolk DA. Alzheimer's Disease Neuroimaging Initiative. Biomarker-based prediction of progression in $\mathrm{MCl}$ : Comparison of $\mathrm{AD}$ signature and hippocampal volume with spinal fluid amyloid- $\beta$ and tau. Front Aging Neurosci 2013; 5: 55

13 Jack CR Jr, Holtzman DM. Biomarker modeling of Alzheimer's disease. Neuron 2013; 80 (6): 1347-1358

14 Risacher SL, Saykin AJ. Neuroimaging and other biomarkers for Alzheimer's disease: the changing landscape of early detection. Annu Rev Clin Psychol 2013; 9: 621628

15 Schulz M, Bohlken J, Hering $R$ et al. Diagnostische und therapeutische Leistungsdichte von neu erkrankten, zu Hause lebenden Patienten mit Demenz. Berlin: Zentralinstitut für die kassenärztliche Versorgung in Deutschland (Zi) - Versorgungsatlas; 2014, http://www.versorgungsatlas.de/themen/alle-analysen-nach-datum-sortiert/?tab=6\&uid=48 (Zugang am 25.02.2015)

16 Kalbe E, Kessler J, Calabrese P et al. DemTect: a new, sensitive cognitive screening test to support the diagnosis of mild cognitive impairment and early dementia. Int J Geriat Psychiatry 2004; 19: 136-143

17 Kessler J, Markowitsch HJ, Denzler P. Mini-Mental-Status-Test (MMST). Göttingen: Beltz Test GMBH; 2000, [Deutsche Adaption]

18 Beck AT, Brown GK et al. Beck-Depressions-Inventar-FS (BDI-FS). Manual. Deutsche Bearbeitung von Sören Kliem \& Elmar Brähler: Pearson Assessment: Frankfurt am Main; 2013

19 Yesavage JA, Brink TL et al. Development and validation of a geriatric depression screening scale: a preliminary report. J Psychiatr Res 1983; 39: $37-39$

20 Karlawish J, Rubright J, Casarett D et al. Older adults' attitudes toward noncompetent subjects participating in Alzheimers research. Am J Psychiatry 2009; 166: $182-188$

21 Boustani M, Perkins AJ, Fox $C$ et al. Who refuses the diagnostic assessment for dementia in primary care? Int J Geriatr Psychiatry 2006; 21: $556-563$

22 Schmidtke K. Lumbalpunktion in der Demenzabklärung: Die Liquorpunktion ist unentbehrlich. Kontra 2014; 85: 478-479
23 Schulz JB. Lumbalpunktion in der Demenzabklärung: Die Liquorpunktion ist unentbehrlich. Nervenarzt 2014; 85: 476-477

24 Luck T, Luppa M, Sieber J et al. Attitudes of the German general population toward early diagnosis of dementia-results of a representative telephone survey. PLoS One 2012; 7: e50792. DOI: 10.1371/journal. pone.0050792

25 Galvin JE, Fu Q Nguyen JT. Psychosocial determinants of intention to screen for Alzheimer's disease. Alzheimers Dement 2008; 4: 353-360

26 Fowler NR, Boustani MA, Frame A et al. Effect of patient perceptions on dementia screening in primary care. J Am Geriatr Soc 2012; 60: 1037 1043

27 Justiss MD, Boustani M, Fox $C$ et al. Patients' attitudes of dementia screening across the Atlantic. Int J Geriatr Psychiatry 2009; 24: 632 637

28 Robinson SM, Canavan M, O'Keeffe ST. Preferences of older people for early diagnosis and disclosure of Alzheimer's disease (AD) before and after considering potential risks and benefits. Arch Gerontol Geriatr 2014; 14: S0167-S0177

29 Kaduszkiewicz H, Eisele M, Wiese B et al. Study on Aging, Cognition, and Dementia in Primary Care Patients (AgeCoDe) Study Group.Prognosis of mild cognitive impairment in general practice: results of the German AgeCoDe study. Ann Fam Med 2014; 12: 158 - 165

30 Reiber H, Lange P. Zerr I Neurochemical Dementia Diagnostics - Inerlaboratory Variation of Analysis, Reference Rnges and Interpretations. J Alzheimers Dis Parkinsonims 2014; 4: 147-156

31 Visser $P$ et al. Disclosure of Alzheimer's disease biomarker status in subjects with mild cognitive impairment. Biomarkers Med 2012; 6 : $1263-1269$

32 Clionsky M, Clionsky E. Dementia Screening: Saying No to the USPSTF and Yes to Brief Cognitive Evaluation. J Alzheimers Dis Parkinsonism 2014; 4: e132

33 Ballard C, Bannister C, Solis $M$ et al. The prevalence, associations and symptoms of depression amongst dementia sufferers. J Affect Disord 1996; 36: $135-144$ 